Supporting Information

\title{
Self-Assembled Polysaccharide- Diphenylalanine/Au Nanospheres for Photothermal Therapy and Photoacoustic Imaging
}

Kaiwen Shen, ${ }^{\dagger}$ Yuting Huang,${ }^{\dagger}$ Qiuju $\mathrm{Li},{ }^{\ddagger}$ Min Chen ${ }^{*}{ }^{\dagger}$ and Limin $\mathrm{Wu}^{\dagger}$

$\dagger$ Department of Materials Science and State Key Laboratory of Molecular Engineering of Polymers, Fudan University, Shanghai 200433, People's Republic of China

\$ State Key Laboratory of Pollution Control and Resource Reuse, College of

Environmental Science and Engineering, Tongji University, Shanghai 200092, People's Republic of China

*E-mail: chenmin@fudan.edu.cn 

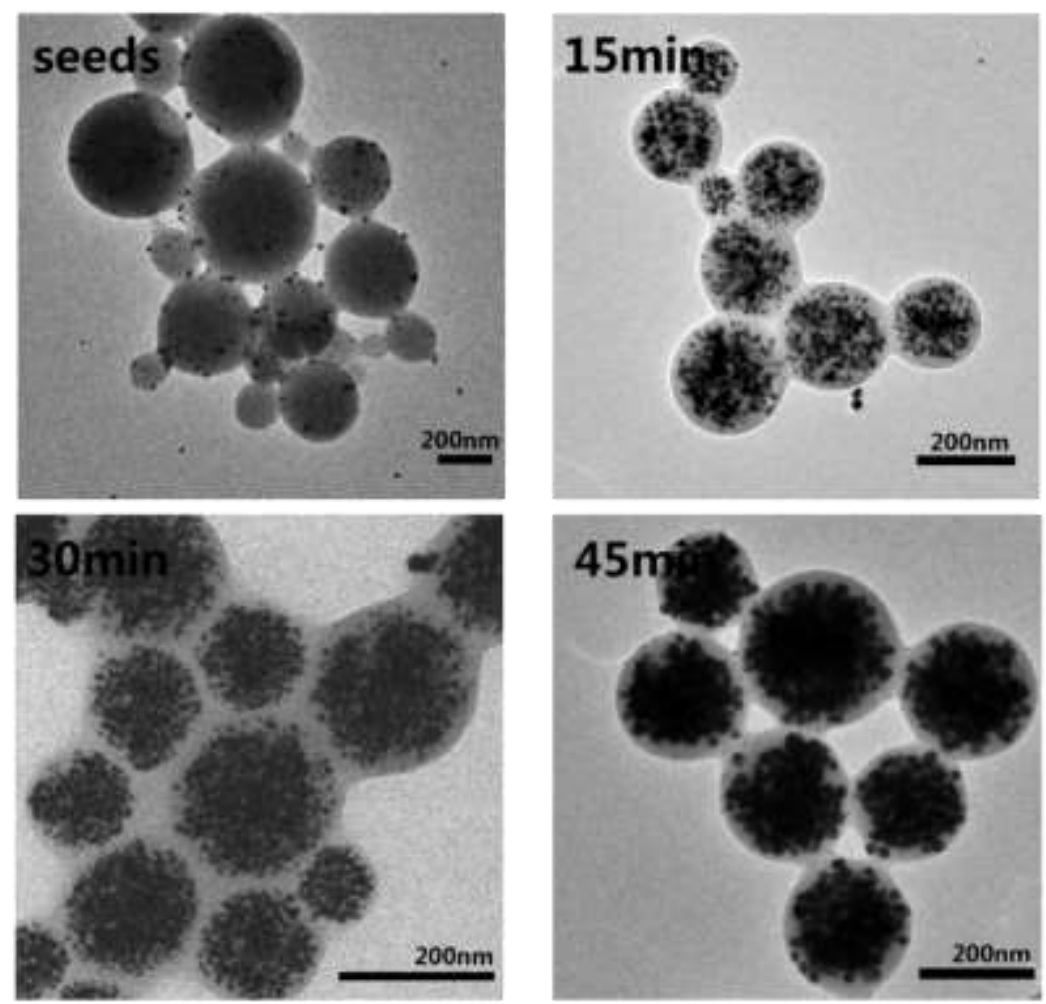

Figure S1. TEM images of ADA-FF/Au nanospheres at different periods while synthesis.

(a)

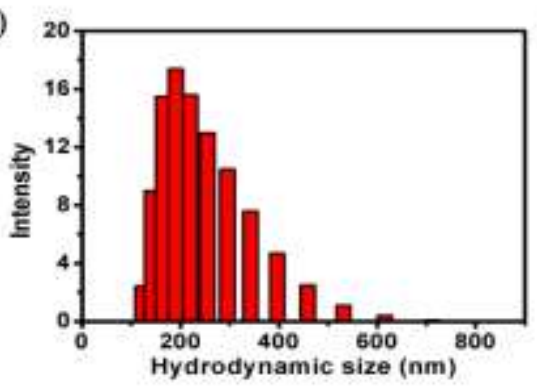

(b)

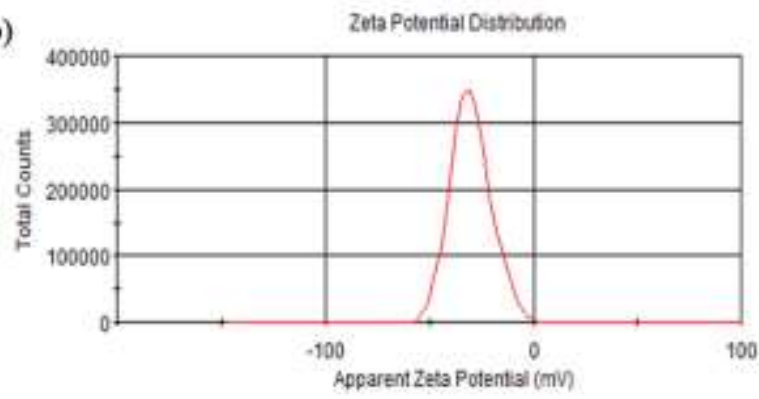

Figure S2. (a) Hydrodynamic sizes (b) and zeta potential of ADA-FF/Au nanospheres. 


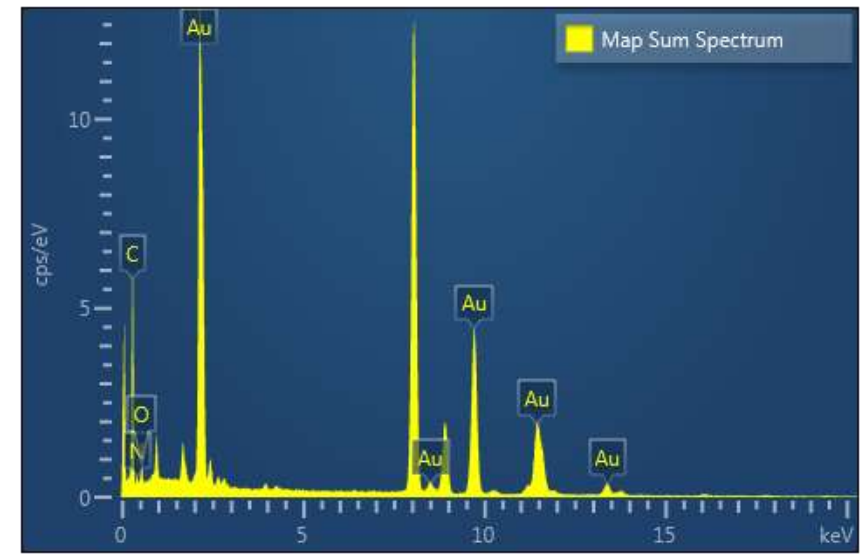

Figure S3. Energy-dispersive X-ray spectroscopy (EDXS) of ADA-FF/Au nanospheres.

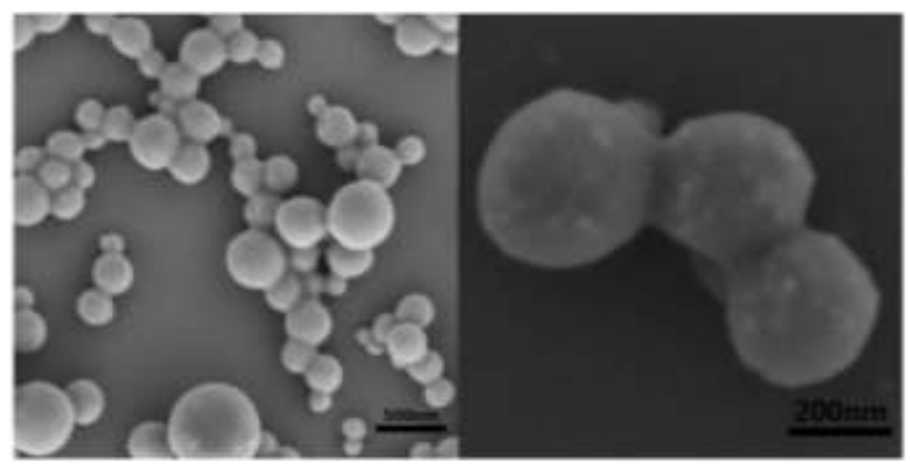

Figure S4. SEM images of ADA-FF/Au nanospheres. 


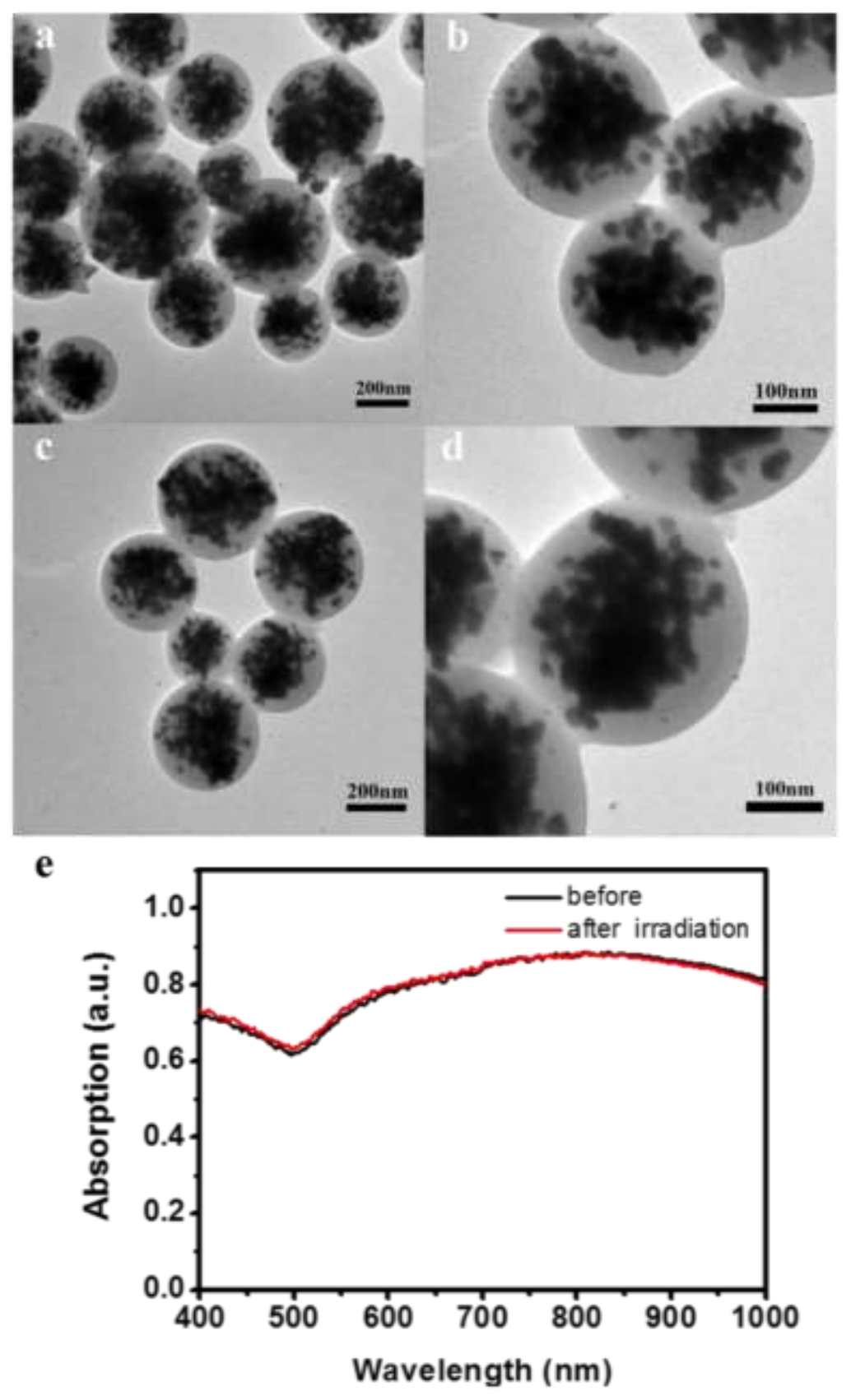

Figure S5. Photothermal stability of ADA-FF/Au nanospheres. TEM images of the sample before $(a, b)$ and after (c. d) being irradiated under $808 \mathrm{~nm}$ laser for 15 minutes. (e) Corresponding absorption spectra. 